\title{
A novel design of algorithm and framework for decentralized CFAR signal detection without prior information
}

\author{
Nan Wang ${ }^{*}$, Yunshan Xu, Haibao Xia, and Jundi Wang \\ Air Force Engineering University, Xi' an 710038, China
}

\begin{abstract}
In this paper, a fusion detection algorithm that focuses on decentralized CFAR (Constant False Alarm Rate) signal detection problem without prior information is proposed. In the algorithm, the threshold and test statistic of the detection fusion algorithm derive from the conventional CFAR detection method. At last a framework for decentralized CFAR signal detection is designed corresponding to the fusion algorithm. Simulation results illustrate that an almost optimal detection performance is obtained by the proposed algorithm.
\end{abstract}

\section{Introduction}

It is a significant problem to fulfil detection in distributed information fusion system. This problem must be up against no matter in multi-static radar or other multi-sensor cooperation detection system [1-3], in which what information to transport and how to design the framework is the key in the system.

Conventional method makes the detection fusion with the entire detectors' result in which a little quantity of information can be used. So it restricts the detection centre's performance [4-7]. As [8] combines the detection results of each detector, and optimize the weight coefficients for any given knowledge to improve the detection performance. With the development of communication ability, a means of double threshold detection arises for distribution detection [9-11]; it must be known that the signal noise rate (SNR) or the detection performance of every detector for reaching the optimal performance [12-14]. If making fusion information directly without the prior knowledge (SNR), the result must lead to a decrease performance for the entire system.

According to the information theory, every detection practice has a positive information in average, so the fusion with decreasing performance shouldn't be applied [15-16]. Of course there are some detection fusion methods without prior information. Reference [17] supposes the prior information deciding the weight of the each detector's detection result, in which the weight is confirmed by probability distribution. Although its performance is below the optimal obviously, the stability of this method is well. On the whole, the number of article is rather less and their performance is unsatisfying in the detection fusion without prior information.

\footnotetext{
*Corresponding author: wang_mn@163.com
} 
This paper puts forward a detection fusion algorithm without prior information. It's significant to make the research for the decentralize detection.

\section{Decentralize CFAR Detection Fusion Algorithm}

\subsection{False Alarm Likelihood Function (FALF)}

According to CFAR threshold detection criterion, assuming judgment method as $\left(x_{1}\right.$ is the test statistic):

$$
\left\{\begin{array}{l}
H_{1}: x_{1}>t h \\
H_{0}: x_{1}<t h
\end{array}\right.
$$

We set the test statistic $x$ 's FALF defined:

$$
P_{f}\left(x_{1}\right)=\int_{x_{1}}^{\infty} p\left(x \mid H_{0}\right) d x
$$

We can get that each test statistic $x$ is corresponding a certain FALF $P_{f}(x)$. According to aforementioned article, if set the $P_{f}\left(x_{1}\right)$ as a new test statistic index, the detection is equal to:

$$
\left\{\begin{array}{l}
H_{1}: P_{f}\left(x_{1}\right)<P_{f a} \\
H_{0}: P_{f}\left(x_{1}\right)>P_{f a}
\end{array}\right.
$$

Next the decentralize detection fusion algorithm based on FALF will be introduced:

\subsection{Detection Fusion Algorithm}

As the aforementioned description, supposing $\mathrm{C}=P_{f a}$, when $P_{f}\left(x_{1}\right)<\mathrm{C}$, the algorithm return $H_{1}$, else return $H_{0}, \mathrm{C}$ is connected only with $P_{f a}$. The judgment correspond to the NeymanPearson criterion.

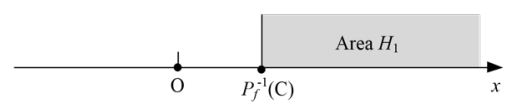

Fig. 1. Single detector.

$P_{f}^{-1}$ is the inverse function to the FALF (figure 1). Then considering double element detection fusion problem, each receive signal's FALF set as: $P_{1 f}\left(x_{1}\right)=\int_{x_{1}}^{\infty} p_{1}\left(x \mid H_{0}\right) d x \quad \& \quad P_{2 f}\left(x_{2}\right)=\int_{x_{2}}^{\infty} p_{2}\left(x \mid H_{0}\right) d x \quad$ When $P_{1 f}\left(x_{1}\right) P_{2 f} \quad\left(x_{2}\right)<\mathrm{C}$, the algorithm return $H_{1}$, else $H_{0}$. C is a function only connected with $P_{f a}$, assuming $\Omega$ (figure 2 : Area $\left.H_{1}\right)$ is the area of $P_{1 f}\left(x_{1}\right) P_{2 f}\left(x_{2}\right)<\mathrm{C}$, and different detector is independent with each other. So the expression of $P_{f a}$ is offered as next integration:

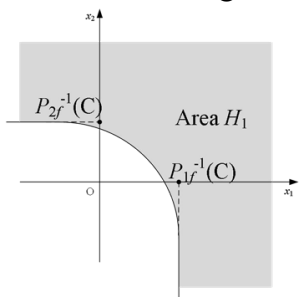

Fig. 2. Double element detector.

$$
P_{f a}=\int_{\Omega} p_{1}\left(x_{1} \mid H_{0}\right) \cdot p_{2}\left(x_{2} \mid H_{0}\right) d x_{1} d x_{2}
$$

The relation between $P_{f a}$ and $\mathrm{C}$ is $P_{f a}=\mathrm{C}(1-\operatorname{lnC})$ (Appendix A), $0<\mathrm{C}<1$. When $P_{f a}$ is a constant, the $\mathrm{C}$ is a constant too. In this way, we get the detection probability $\left(P_{d}\right)$ as:

$$
P_{d}=\int_{\Omega} p_{1}\left(x_{1} \mid H_{1}\right) \cdot p_{2}\left(x_{2} \mid H_{1}\right) d x_{1} d x_{2}
$$


Next a generalize method is introduced for $N$ element detection fusion, supposing the FALF of $i^{\text {th }}$ detector receiving signal is defined as:

$$
P_{i f}\left(x_{i}\right)=\int_{x_{i}}^{\infty} p_{i}\left(x \mid H_{0}\right) d x
$$

When $\prod_{i=1}^{N} P_{i f}\left(x_{i}\right)<C$, the fusion system return $H_{1}$, else return $H_{0}$, as the aforementioned paper, assuming $\Omega$ is the area of $H_{1}$, so the $P_{f a}$ as:

$$
P_{f a}=\int_{\Omega} \prod_{i=1}^{N}\left[p_{i}\left(x_{i} \mid H_{0}\right)\right] d x_{1} \cdots d x_{N}
$$

The relation between $P_{f a}$ and $C$ is $P_{f a}=C \cdot \sum_{i=0}^{N-1} \frac{(-\ln C)^{i}}{i !}$ (Appendix B), $0<C<1$. When $P_{f a} \& N$ is confirmed, the $\mathrm{C}$ is confirmed too. So $\mathrm{C}$ is the function of $P_{f a}$ and $N($ the number of element for detection fusion). In this way, we get the detection probability $\left(P_{d}\right)$ as:

$$
P_{d}=\int_{\Omega} \prod_{i=1}^{N}\left[p_{i}\left(x_{i} \mid H_{1}\right)\right] d x_{1} \cdots d x_{N}
$$

When making an $N$ element detection fusion, the $\mathrm{C}$ should be got firstly, and then the judgment is made by comparing the product of $N$ FALF and $\mathrm{C}$. We offer a series of $\mathrm{C}$ with the change of the $P_{f a}$ in multi-element detection fusion as figure 3 and table 1:

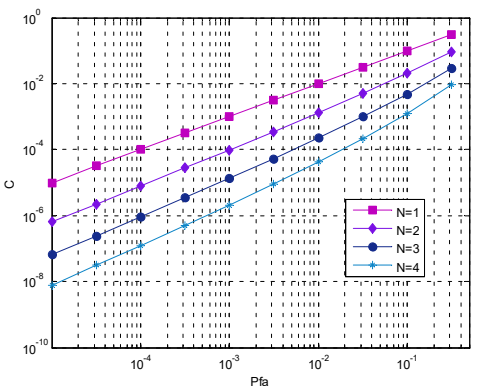

Fig. 3. The relation among $\mathrm{C}$ and $\mathrm{P}_{f a} \& \mathrm{~N}$.

Table 1. The value of $C$ when confirming $\mathrm{P}_{f a} \& N$.

\begin{tabular}{|c|c|c|c|c|}
\hline $\mathrm{P}$ & 1 & 2 & 3 & 4 \\
\hline $10^{-1}$ & $10^{-1}$ & $2.05 \times 10^{-2}$ & $4.88 \times 10^{-3}$ & $1.25 \times 10^{-3}$ \\
\hline $10^{-2}$ & $10^{-2}$ & $1.31 \times 10^{-3}$ & $2.24 \times 10^{-4}$ & $4.34 \times 10^{-5}$ \\
\hline $10^{-3}$ & $10^{-3}$ & $9.77 \times 10^{-5}$ & $1.33 \times 10^{-5}$ & $2.12 \times 10^{-6}$ \\
\hline $10^{-4}$ & $10^{-4}$ & $7.84 \times 10^{-6}$ & $8.93 \times 10^{-7}$ & $1.23 \times 10^{-7}$ \\
\hline
\end{tabular}

\section{The Detection Framework Based on The Algorithm}

\subsection{Centralize Detection Framework}

Centralize framework is efficient for detection fusion [2]; the structure as the figure 4:

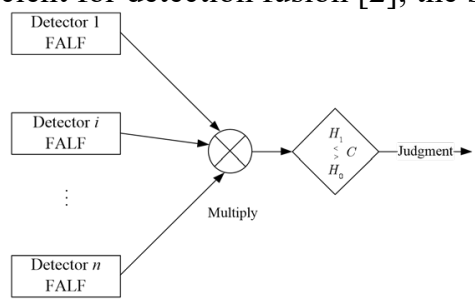

Fig. 4. Structure of centralize detection. 
From the figure 4, every detector transmits its FALF rather than the judgment or initial signal and the fusion centre make the judgment through a product calculation. According to the framework design the algorithm as table 2:

Table 2. Algorithm Process.

\begin{tabular}{|l|l|}
\hline import & $\begin{array}{l}\text { The false alarm probability } P_{f a} \\
\text { The Receiving signal } x_{i} \text { of detector } i\end{array}$ \\
\hline algorithm & $\begin{array}{l}\text { 1. } \quad \text { Calculate the FALF of } x_{i} \\
\end{array}$ \\
& 2. $\quad$ Get the value of C relying on $P_{f a} \& \mathrm{~N}$ \\
\hline export & Detection result \\
\hline
\end{tabular}

\subsection{Sequential Detection Construction}

The previous detection framework is a rather rigid structure such as some detector in breakdown. So it is essential to design a flexible structure for realistic environment. Designing a sequential detection framework as figure 5 :

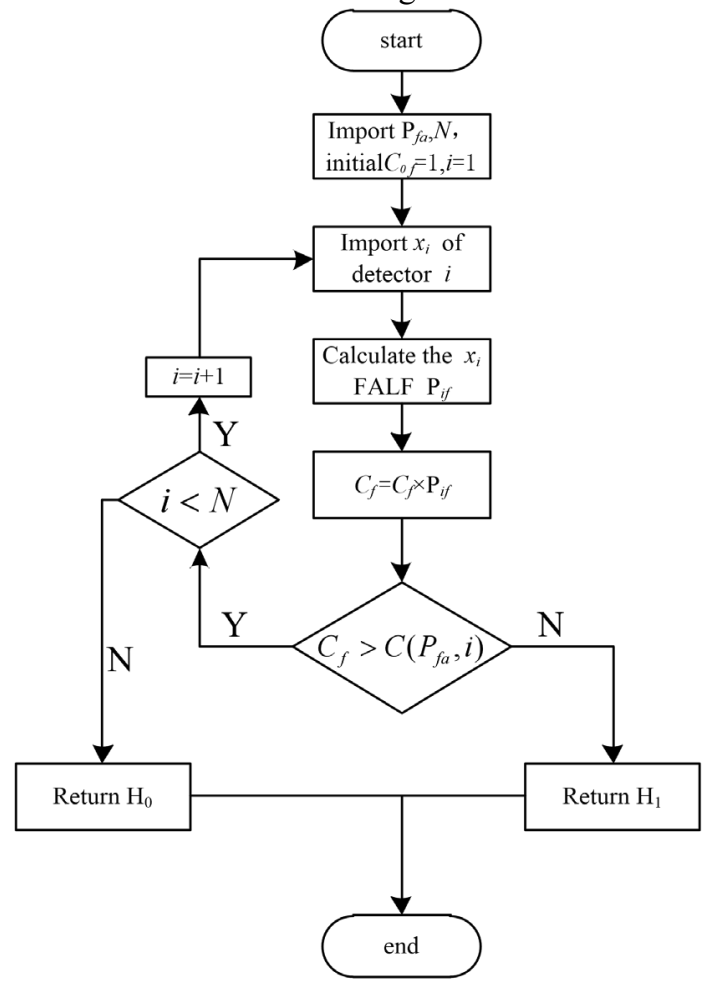

Fig. 5. Sequential detection fusion algorithm structure.

When making the judgment of $H_{1}$, it is no need to fusion all the element detection actually. Only if product of part element's FALF reaches the threshold, can the judgment be made. In this way, the system can still fulfil the detection if some detector is missed.

\subsection{Adaptive Threshold Detection Framework}

Design an adaptive detection structure in which each detector's threshold is based on other detector's FALF. The structure is shown as figure 6: 


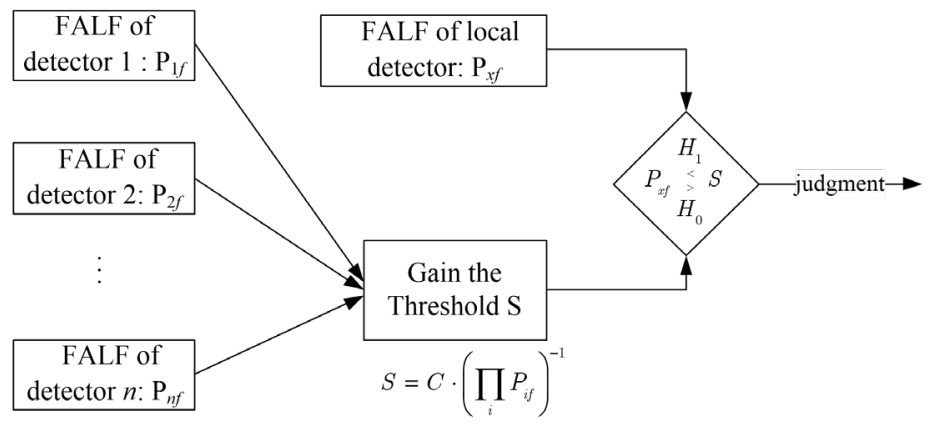

Fig. 6. Adaptive threshold detection.

This framework shows that each detector's threshold will be adjusted after receiving FALF from other detector, and guarantee the entire system's CFAR characteristic. This method will get the same detection performance as the centralize method in 3.1. The communication is essential to make interaction in all detectors in this structure. And algorithm should refer to the previous indicate and adjust the part of corresponding threshold.

\section{Simulation and Analysis}

\subsection{The Detection under Gaussian Distribution}

Set the detector's detection SNR, set detector 2's SNR reach from $-5 \mathrm{~dB}$ to $8 \mathrm{~dB}$ as the base, the detector 1 less $1.5 \mathrm{~dB}$ than the base, the detector 3 more $1.5 \mathrm{~dB}$. Set the $P_{f a}=10^{-2}$. The optimal detection curve is the Bayes method result with the prior SNR information, the result as figure $7 \&$ figure 8 :

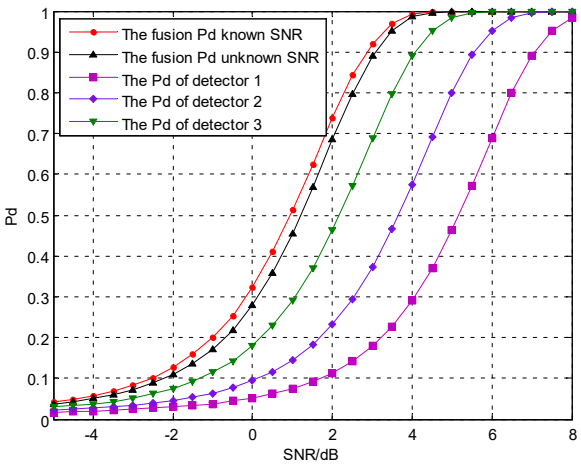

Fig. 7. The $\mathrm{P}_{d}$

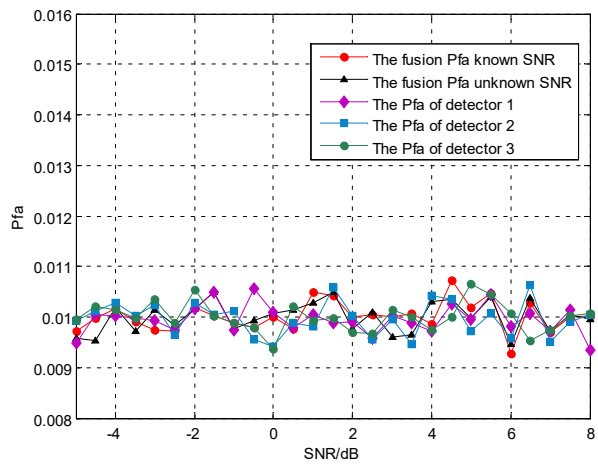

Fig. 8. The $\mathrm{P}_{f a}$.

When fusion detection $P_{d}$ reaches from $0.5---0.9$ by the algorithm of this paper, each performance of detector and the fusion $\mathrm{P}_{d}$ with known SNR by Bayes detection as table 3,

Table 3. The value of $\mathrm{P}_{d .}$.

\begin{tabular}{|c|c|c|c|c|c|}
\hline & \multicolumn{5}{|c|}{$\mathrm{P}_{d}$} \\
\hline The fusion $\mathrm{P}_{d}$ with unknown SNR & 0.5 & 0.6 & 0.7 & 0.8 & 0.9 \\
\hline The $\mathrm{P}_{d}$ of detector 1 & 0.08 & 0.10 & 0.12 & 0.15 & 0.19 \\
\hline The $\mathrm{P}_{d}$ of detector 2 & 0.16 & 0.20 & 0.24 & 0.29 & 0.39 \\
\hline The $\mathrm{P}_{d}$ of detector 3 & 0.32 & 0.41 & 0.49 & 0.58 & 0.71 \\
\hline The fusion $\mathrm{P}_{d}$ with known SNR & 0.56 & 0.67 & 0.76 & 0.84 & 0.93 \\
\hline
\end{tabular}


After Monte Carlo simulation, the detection algorithm is proved a CFAR method. The performance of this method is close to the optimal from the simulation.

\subsection{The Detection under Rice Distribution}

Next we make the simulation the detection fusion under the Rice distribution. It is a common distribution in an envelope detector. Set two orthogonal signals of every detector in which each signal's SNR agree with the 4.1 chapter in the corresponding detector. The simulation result as figure $9 \&$ figure 10 :

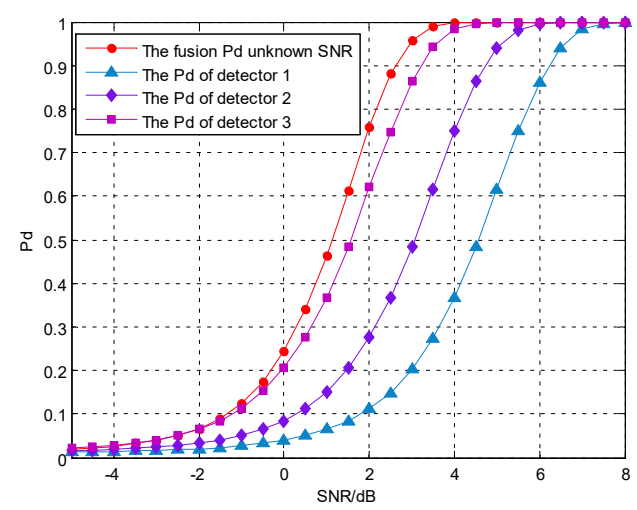

Fig. 9. The $\mathrm{P}_{d}$.

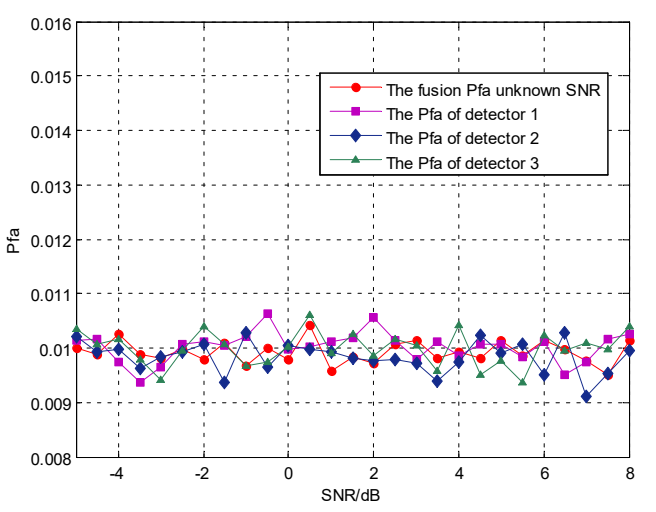

Fig. 10. The $\mathrm{P}_{f a}$.

When $P_{d}$ reach from 0.5 ---0.9, each performance of detector as table 4.

Table 4. The value of $\mathrm{P}_{d}$.

\begin{tabular}{|c|c|c|c|c|c|}
\hline The fusion $\mathrm{P}_{d}$ with unknown SNR & 0.5 & 0.6 & 0.7 & 0.8 & 0.9 \\
\hline The $\mathrm{P}_{d}$ of detector 1 & 0.07 & 0.08 & 0.10 & 0.12 & 0.16 \\
\hline The $\mathrm{P}_{d}$ of detector 2 & 0.16 & 0.20 & 0.24 & 0.30 & 0.39 \\
\hline The $\mathrm{P}_{d}$ of detector 3 & 0.39 & 0.47 & 0.56 & 0.67 & 0.82 \\
\hline
\end{tabular}

The fusion algorithm performs a better detection performance than each detector.

\section{Conclusion}

In this paper, a novel detection fusion method without prior information is proposed after the analysis of problem in decentralizes detection, which test statistic is from the FALF of receiving signal. And several frameworks for the fusion are offered. Simulation results illustrate that an almost optimal detection performance is obtained by the proposed algorithm. In which the optimal detection fusion performance is the Bayes method result with the prior SNR information. Although only the threshold detection is researched in the paper, we can transform the non-threshold detection into the threshold detection. Moreover, the further work should offer the optimal detection method in the Rice distribution.

\section{Appendices}

\subsection{Appendix A}


considering: $\quad P_{f a}=\int_{\Omega} p_{1}\left(x_{1} \mid H_{0}\right) p_{2}\left(x_{2} \mid H_{0}\right) d x_{1} d x_{2}, \Omega$ is the area of $P_{1 f}\left(x_{1}\right) P_{2 f}\left(x_{2}\right)<C$, supposing $P_{1 f}\left(x_{1}\right)=\int_{x_{1}}^{\infty} p_{1}\left(x \mid H_{0}\right) d x$ and $P_{2 f}\left(x_{2}\right)=\int_{x_{2}}^{\infty} p_{2}\left(x \mid H_{0}\right) d x$ 。

Proof:

$$
\begin{aligned}
P_{f a} & =\int_{\Omega} p_{1}\left(x_{1} \mid H_{0}\right) \cdot p_{2}\left(x_{2} \mid H_{0}\right) d x_{1} d x_{2} \\
& =\int_{-\infty}^{P_{2 f}^{-1}(C)} p_{2}\left(x_{2} \mid H_{0}\right) \int_{f\left(x_{2}\right)}^{\infty} p_{1}\left(x_{1} \mid H_{0}\right) \cdot d x_{1} d x_{2}+\int_{P_{2 f}^{-1}(C)}^{\infty} p_{2}\left(x_{2} \mid H_{0}\right) d x_{2} \\
& =\int_{-\infty}^{P_{2 f}^{-1}(C)} p_{2}\left(x_{2} \mid H_{0}\right) \frac{C}{P_{2 f}\left(x_{2}\right)} d x_{2}+C \\
& =-\left.C \cdot \ln \left[P_{2 f}\left(x_{2}\right)\right]\right|_{-\infty} ^{P_{2 f}^{-1}(C)}+C \\
& =C(-\ln C+1)
\end{aligned}
$$

Because of $C \in(0,1)$, When $\mathrm{P}_{f a}$ is a constant, the $C$ is a constant too.

\subsection{Appendix B}

Proof:

$$
\begin{aligned}
& P_{f a}=\int_{\Omega} \prod_{i=1}^{N}\left[p_{i}\left(x_{i} \mid H_{0}\right)\right] d \Omega=C \cdot \sum_{i=0}^{N-1} \frac{(-\ln C)^{i}}{i !}, \Omega \text { is the area of } \prod_{i=1}^{N} P_{i f}\left(x_{i}\right)<C, \\
& P_{i f}\left(x_{i}\right)=\int_{x_{i}}^{\infty} p_{i}\left(x \mid H_{0}\right) d x, \text { supposing } f_{N}(C)=C \cdot \sum_{i=0}^{N-1} \frac{(-\ln C)^{i}}{i !}
\end{aligned}
$$

Mathematical Induction: when $\mathrm{N}=1$, assuming $\mathrm{N}=\mathrm{k}$ it is true too, to calculate the formula when $\mathrm{N}=\mathrm{k}+1$ :

$$
\begin{aligned}
P_{f a} & =\int_{\Omega} \prod_{i=1}^{k+1}\left[p_{i}\left(x_{i} \mid H_{0}\right)\right] d \Omega \\
& =\int_{-\infty}^{P_{k f}^{-1}(C)} p_{k+1}\left(x_{k+1} \mid H_{0}\right) \int_{\Omega^{\prime}} \prod_{i=1}^{k}\left[p_{i}\left(x_{i} \mid H_{0}\right)\right] d \Omega^{\prime} d x_{k+1}+C \\
& =\int_{-\infty}^{P_{k f}^{-1}(C)} p_{k}\left(x_{k} \mid H_{0}\right) f_{k}\left(\frac{C}{P_{k f}\left(x_{k}\right)}\right) d x_{k}+C \\
& =C \cdot\left(\int_{1}^{C} f_{k}(t) \cdot \frac{1}{t^{2}} d t+1\right) \\
& =C \cdot\left[-\sum_{i=0}^{k-1} \frac{(-1)^{i}(\ln C)^{i+1}}{(i+1) !}+1\right] \\
& =C \cdot \sum_{i=0}^{k} \frac{(-\ln C)^{i}}{i !}
\end{aligned}
$$

\section{Acknowledgments}

This research was funded by the Science and Technology on Avionics Integration

Laboratory \& Aeronautical Science Fund (20155596024). 


\section{References}

1. Ramanarayanan Viswanathan, Pramod K. Varshney. Distributed Detection With Multiple Sensors: Part I—Fundamentals [J]Proceedings of the IEEE, 1997 85(1),54-63

2. Mahesh K. Banavar, Anthony D. Smith, Cihan Tepedelenlioglu, Andreas Spanias. On the Effectiveness of Multiple Antennas in Distributed Detection over Fading MACs. [J]. IEEE Transactions on Wireless Communications ,2012, 11(5): 1744-1452

3. Alla Tarighati, James Grossy, Joakim Jalden. Decentralized Hypothesis Testing in Energy Harvesting Wireless Sensor Networks [J] IEEE Transactions on signal processing ,2017, 65(18): 4862-4873

4. Hang Rao, Xiaojing Shen, Yunmin Zhu and Jianxin Pan. Distributed Detection Fusion via Monte Carlo Importance Sampling[C] Chinese Control Conference. 2016: 48304835

5. Y Li, DK Jha, A Ray, TA Wettergren. Information Fusion of passive sensors for detection of moving targets in Dynamic environments [J] IEEE Transactions on Cybernetics, 2016,47(1):93-104.

6. D. Ciuonzo, P. Salvo Rossi. Decision Fusion with Unknown Sensor Detection Probability.[J] IEEE Signal Processing Letters 2014,21(2):208-212

7. D. Ciuonzo, P. Salvo Rossi, P. Willett. Generalized Rao Test for Decentralized Detection of an Uncooperative Target[J] IEEE Signal Processing Letters, 2017,24(5):678-682.

8. Hong-Yan Zhao, Jun Liu, Zi-Jing Zhang, Hongwei Liu, Shenghua Zhou. Linear Fusion for Target Detection in Passive Multistatic Radar[J] Signal Processing,2016, 130@: $175-182$.

9. HU Qinzhen SU Hongtao ZHOU Shenghua LIU Ziwe. Double Threshold CFAR Detection for Multisite Radar. [J] Journal of Electronics\& Information Technology, 2016,38(10):2430-2436, Chinese.

10. Ishrat Maherin, Qilian Liang. Multistep Information Fusion for Target Detection Using UWB Radar Sensor Network[J] IEEE Sensors Journal, 2015,15(10):5927-5937.

11. Aleksandar Jeremi'c, Kon Max Wong, Bin Liu. Optimal Distributed Detection of Multiple Hypotheses using Blind Algorithm[C]Conference Paper in IEEE Transactions on Aerospace and Electronic Systems, 2009,47(1):317-331.

12. Sungho Kim. High-Speed Incoming Infrared Target Detection by Fusion of Spatial and Temporal Detectors [J]Sensors 2015, 15(4): 7267 -7293.

13. Guoliang Zhang, Chunling Yang, Yan Zhanga, Yang Jiao. A Novel Approach based on Spectral-Temporal Information Fusion for Multi-Target Detection[C] IEEE 11th Conference on Industrial Electronics and Applications (ICIEA) 2016:1661-1665.

14. Jing Ni, Jie Mei. A Fusion Algorithm for Target Detection in Distributed Sensor Networks[C] Sixth International Conference on Computational Intelligence and Communication Networks, 2014:349-353

15. Wee Peng Tay. The Value of Feedback in Decentralized Detection.[J] IEEE Transactions on information theory, 2012, 58(12):7226-7239

16. Alla Tarighati, Joakim Jalden. Rate Allocation for Decentralized Detection in Wireless Sensor Networks[C] IEEE International Workshop on Signal Processing Advances in wireless Communication, 2015,7405(s 1-3):479-487. 
17. Edmond Nurellari, Sami Aldalahmeh, Mounir Ghogho1, Des McLernon. Quantized Fusion Rules for Energy-Based Distributed Detection in Wireless Sensor Networks [C] sensor signal processing for defence, 2014:1-5. 